\title{
The punctal apposition syndrome: a new surgical approach
}

\section{C Francis, M K Wan}

Br J Ophthalmol 2002;86:1256-1258

Aims: To assess the punctal apposition syndrome (PAS) and its response to lateral canthal tendon (LCT) repair. Methods: In this retrospective, interventional case series, five patients (seven symptomatic eyes) with PAS were managed. Lateral canthal tendon repair was performed in all seven eyes. The main outcome measure was correction of watery eye symptomatology.

Results: All five patients achieved symptomatic resolution. Conjunctivochalasis and functional nasolacrimal duct obstruction were associated with the PAS.

Conclusion: This new surgical approach to the PAS, using a LCT repair, was successful in all patients. Two patients (three eyes) required conjunctivochalasis excision.

E piphora is a common condition. Bartley ${ }^{1}$ classified the causes of acquired lacrimal drainage obstruction as primary and secondary. The rare condition of "kissing puncta $^{\prime 12}$ represents the clinical abnormality in the punctal apposition syndrome (PAS) and is a secondary mechanical cause of acquired lacrimal drainage obstruction. The PAS represents the situation in which at any stage during the blinking cycle, other than when the puncta actively appose during the closure phase of blinking, the puncta are apposed and the patient experiences watering.

This study reports a series of five patients (seven eyes) with PAS and discusses its active surgical treatment. We have described a new approach to the surgical treatment of PAS utilising the well established lateral tarsal strip procedure, ${ }^{34}$ which we have labelled the lateral canthal tendon (LCT) repair.

In 1991, Glatt described management of the PAS in a single patient using a ptosis procedure, which effectively elevated the upper punctum away from the lower. ${ }^{5}$ These apposing puncta were described by Bartley as "kissing puncta." ${ }^{1}$ Hurwitz, in his definitive 1996 text, also implied that correcting ptosis was the most appropriate method of treating PAS. ${ }^{2}$ We undertook this study to assess the clinical features of five patients with PAS, and the outcome of management using LCT repair. To our knowledge, there has been no previous case series describing the management of PAS.

\section{METHODS}

Five patients (seven eyes) were seen in a specialist ocular plastics unit. A full ocular plastics history and examination were performed, ${ }^{6}$ noting whether the patients had true epiphora or plerolacrima. We define plerolacrima ${ }^{7}$ as the patient having symptomatic watery eyes, but without tears running down the face. In epiphora, patients complain of tears running down their cheek. This is expanded in the discussion.

Examination of the face, lids, state, and position of the puncta and everted eyelid appearance was performed. In particular, the position of the lower punctum in relation to its normal location at the very lateral border of the plica semilunaris was assessed. Dry eyes and refractive error were excluded. The patients' lid closure and blink reflex were normal. The intactness of the facial nerve was confirmed. The snapback test, ${ }^{2}$ horizontal lid distractibility (medial and lateral $)^{7}$ and Jones testing $\mathrm{I}$ and $\mathrm{II}^{8}$ were performed. To improve sensitivity, Jones testing was augmented by nasal endoscopic examination, using a $2.7 \mathrm{~mm}$ Storz nasal endoscope, to detect definitively the presence of fluorescein under the inferior turbinate. Once the PAS was confirmed as part of the underlying cause of watering and definite laxity of the LCT was established, surgery was offered to the patient.

A definitive LCT repair was performed. In our cases, we augmented it with a periosteal or temporalis fascia flap. ${ }^{79}$

Patients were questioned about their watery eye symptomatology, and relative punctal position assessed.

\section{Surgical technique}

The aim of the LCT repair in surgery for the PAS is to separate the lower punctum from its apposing upper counterpart with the eyes open and in primary gaze, and at the same time, to reposition the lower punctum at the lateral border of the plica semilunaris, its normal anatomical position.

The procedure commences with local infiltration with bupivacaine, lignocaine, and adrenaline at the lateral canthal region in combination with assisted local anaesthesia. ${ }^{10}$ A skin incision is made at the lateral commissure, and the inferior limb of the LCT is identified and divided at or just lateral to the lateral commissure.

A tarsal strip of the appropriate length is fashioned and freshened. It is sutured to the appropriate position on the lateral orbital margin using a double armed 6/0 Ticron tendon-type suture. The tension is adjusted to optimise the lower lid position with respect to the lower punctum in relation to the plica semilunaris. The tarsal strip is reinforced by either a local periosteal or temporalis fascia flap, as we have found this retains the integrity of the new lateral canthal angle. Muscle and skin are closed in layers.

\section{RESULTS}

Five patients with the PAS and watery eyes are described. Ages ranged from 63 to 77 years, with a mean of 72 years. There were three males and two females. Two patients (three eyes) had epiphora and three patients (four eyes) had plerolacrima.

Four patients (six eyes) had conjunctivochalasis. ${ }^{11}$ All patients had Jones 2 testing positive and Jones 1 testing negative, thereby defining them as having a functional nasolacrimal duct obstruction (FNLDO). ${ }^{12}$ No patient had symptomatic ptosis.

All seven eyes underwent augmented LCT repair, and improved symptomatically. Two patients (three eyes) also required conjunctivochalasis excision.

Table I summarises the clinical assessment and the results of surgery. Figure 1 illustrates the typical appearance of the PAS, and Figure 2 demonstrates the abolition of the PAS with 
Table 1 Findings, management, and outcome in PAS

\begin{tabular}{|c|c|c|c|c|c|c|c|}
\hline Case & Sex & Age & Side & Symptoms and duration & Findings & Management & Result and follow up duration \\
\hline 1 & M & 63 & $R, L$ & Plerolacrima, 15 years & $\begin{array}{l}\text { PAS, CC, LCT laxity, Jones } 2 \\
\text { positive }\end{array}$ & CC excision, $\mathrm{LCT}$ repairs & No tearing, 1 month \\
\hline 2 & M & 73 & $\mathrm{R}$ & Plerolacrima, 8 months & $\begin{array}{l}\text { PAS, CC, LCT laxity, Jones } 2 \\
\text { positive }\end{array}$ & CC excision, $\mathrm{LCT}$ repair & No tearing, 2 months \\
\hline 3 & M & 75 & L & Epiphora, 12 months & PAS, CC, Jones 2 positive & LCT repair & Tearing improved, 14 months \\
\hline 4 & $\mathrm{~F}$ & 73 & $\mathrm{~L}$ & Plerolacrima, 15 months & PAS, Jones 2 positive & LCT repair & No tearing, 3 months \\
\hline 5 & $\mathrm{~F}$ & 77 & $R, L$ & Epiphora, 2 years & PAS, CC, Jones 2 positive & LCT repairs & No tearing, 3 months \\
\hline
\end{tabular}

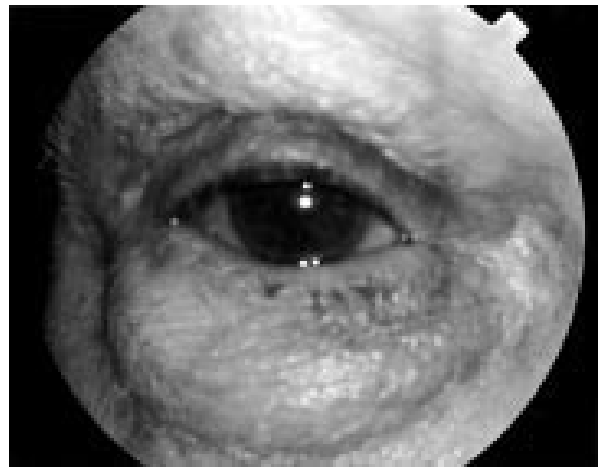

Figure 1 Punctal apposition (right eye).

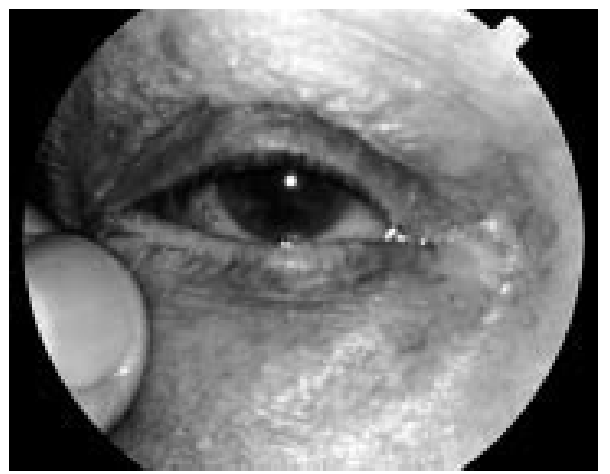

Figure 2 With lateral digital distraction of right lower lid, punctal apposition is abolished.

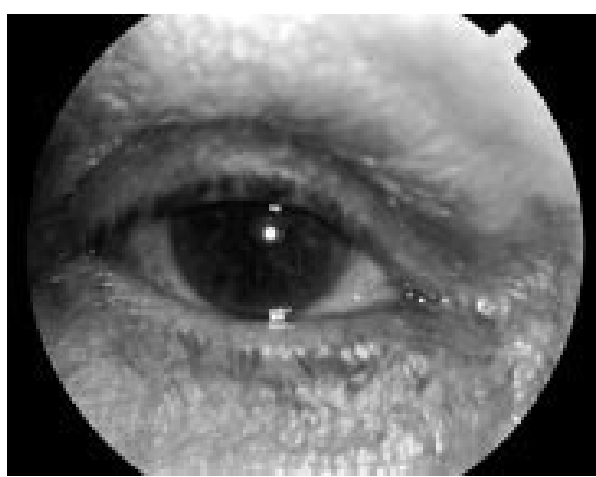

Figure 3 Postoperative view following punctal apposition surgery.

digital lateral distraction of the lower lid. Figure 3 illustrates the postoperative appearance.

\section{DISCUSSION}

Glatt was the first to describe active surgical management of the PAS, in a single case. ${ }^{5}$ Blepharoptosis repair relieved the punctal apposition, thus resolving his epiphora. By contrast, most patients with ptosis do not develop watery eyes, unless the lower lid is also lax and PAS develops as a result.

There is only one other report of the PAS. This occurred in a single patient with Graves' orbitopathy. ${ }^{13}$ In this patient, bilateral transcutaneous orbital fat decompression was done and relieved the patient's PAS and watery eyes symptoms.

We assessed and managed five patients with the PAS. Symptomatic relief of watering was achieved in all cases following surgical intervention using an LCT repair. Success was defined as total relief of watery eye symptomatology in four of our five patients (six eyes) and substantial improvement (of 65\%) in one patient (one eye). Definitive postoperative lacrimal drainage assessment with Jones testing was not performed. While this may be a weakness of our study method, the fact that all patients improved following surgery suggests that the operative technique of LCT repair is beneficial.

All seven eyes operated using an LCT repair had the lower punctum moved to a substantially better anatomical position-that is, where the lower punctum lies lateral to the upper punctum. The time tested operation of an LCT repair offers a new approach to treating the PAS. None of our five patients had symptomatic ptosis, although in the presence of generalised collagen failure (an involutional change) affecting the lids in the older age group, ptosis is not uncommon.

The aetiology of the PAS in our cases is likely to be caused by age related collagen failure of the LCT. ${ }^{14}$ Four of our five patients ( six eyes) also had conjunctivochalasis, ${ }^{11}$ but only two of our patients (three eyes) required conjunctivochalasis excision. ${ }^{15}$ FNLDO was present in four patients (six eyes). This was an interesting finding, as no patient required dacryocystorhinostomy (DCR) surgery, the treatment of choice for FNLDO. ${ }^{12}$ A possible explanation for the high frequency of FNLDO in the PAS is that LCT laxity, which is probably the main factor in the PAS, reduces the effective action of the active lacrimal pump on the lacrimal sac fascia, ${ }^{8}$ producing less negative pressure during the closure phase of each blink. Thus, a relatively loose LCT may allow the lower lid to destabilise and move excessively medially during each blink, resulting in decreased distention of the lacrimal sac.

\section{Plerolacrima}

Patients with epiphora (Greek $=$ addition) experience tears overflowing onto the face. We have coined the term "plerolacrima" $($ Plero $=$ full of (Greek), lacrima = tears (Latin)). Patients with plerolacrima describe symptomatic watering, but their tears almost never run over onto their cheeks. They complain of every symptom that patients with epiphora experience, including blurring of vision, wetness of the eyelids, embarrassment, and general discomfort. Conjunctivochalasis ${ }^{15}$ also produces watery eye symptomatology, which is frequently plerolacrima. It was noteworthy that conjunctivochalasis surgery was combined with LCT repair in two of the three patients who had plerolacrima. 


\section{Conjunctivochalasis}

Conjunctivochalasis was found in four of our five patients (six of seven eyes) and probably also reflects collagen failure. Conjunctivochalasis represents redundancy of the lower lid margin bulbar conjunctiva, which can protrude forwards onto the horizontal lid margin and even further. ${ }^{11}$ Alone, it produces plerolacrima. We felt its severity warranted surgical removal in two patients (three eyes).

In conclusion, our series of patients describes a syndrome of acquired lacrimal drainage obstruction caused by punctal apposition and treated successfully by an LCT repair. However, there appeared to be a contribution by FNLDO and conjunctivochalasis, which may also require treatment in some patients.

\section{Authors' affiliations}

I C Francis, M K Wan, Ocular Plastics Unit, Prince of Wales Hospital and the University of NSW, Sydney, New South Wales 2031, Australia

Correspondence to: Dr lan C Francis, Suite 12, Chatswood Grove, 12-14 Malvern Avenue, Chatswood, NSW 2067, Australia; if@student.unsw.edu.au

Accepted for publication 7 June 2002

\section{REFERENCES}

1 Bartley GB. Acquired lacrimal drainage obstruction: an etiologic classification system, case reports, and a review of the literature. Part 3. Ophthalmic Plast Reconstr Surg 1993;1:11-26.
2 Hurwitz JJ. Examination of the eyelids. In: The lacrimal system. Philadelphia: Lippincott-Raven, 1996:48-9.

3 Anderson RL. The tarsal strip procedure. Arch Ophthalmol 1997; 115:2192-6.

4 Tenzel RR, Buffam FV, Miller GR. The use of the "lateral canthal sling" in ectropion repair. Can J Ophthalmol 1977;12:199-202.

5 Glatt HJ. Epiphora caused by blepharoptosis (Letter). Am J Ophthalmol $1991 ; 111: 649-50$

6 Hurwitz JJ. Examination of the eyelids. In: The lacrimal system. Philadelphia: Lippincott-Raven, 1996:47.

7 Francis IC, Wilcsek GA, Sharma S, et al. Transcaruncular medial orbitotomy for stabilisation of the posterior limb of the medial canthal tendon. Clin Exp Ophthalmol $2001 ; 26: 85-9$.

8 Jones LT. An anatomic approach to problems of the eyelids and lacrimal apparatus. Arch Ophthalmol 1962;66:147-50.

9 Nesi FA, Lisman RD, Levine MR. Smith's ophthalmic plastic and reconstructive surgery. 2nd ed.. Sydney: Mosby, 1998:294

10 Francis IC, Schumacher RS, Haylon M. Assisted local anaesthesia for cataract surgery (ALACS). Aust NZ J Ophthalmol 1987;15:185-91.

11 Liu D. Conjunctivochalasis: a cause of tearing and its management. Ophthalmic Plast Reconstr Surg 1986;2:25-8.

12 Conway ST. Evaluation and management of "functional" nasolacrimal blockage: results of a survey of the American Society of Ophthalmic Plastic and Reconstructive Surgery. Ophthalmic Plast Reconstr Surg 1994; 10:185-7.

13 Cheema MM, Meyer DR. Epiphora secondary to punctal apposition in the setting of Graves' orbitopathy. Ophthalmic Plast Reconstr Surg $1995 ; 11: 122-4$.

14 Shore JW. Changes in lower eyelid resting position, movement, and tone with age. Am J Ophthalmol 1985;99:415-23.

15 Meller D, Tseng SC. Conjunctivochalasis: literature review and possible pathophysiology. Surv Ophthalmol 1998;43:225-32.

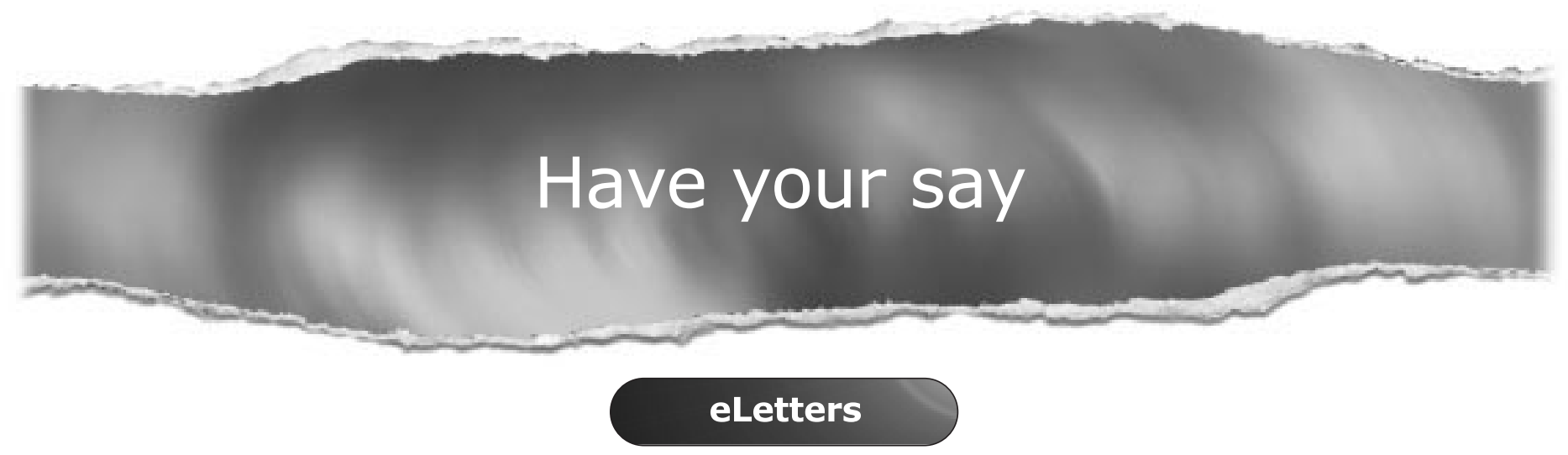

If you wish to comment on any article published in the British Journal of Ophthalmology you can send an eLetter using the eLetters link at the beginning of each article. Your response will be posted on British Journal of Ophthalmology online within a few days of receipt (subject to editorial screening). 